http://jmscr.igmpublication.org/home/ ISSN (e)-2347-176x ISSN (p) 2455-0450 crossref DOI: https://dx.doi.org/10.18535/jmscr/v8i7.14

\title{
Submental Liposuction: 30 Cases Review
}

\author{
Authors \\ Dr Supriya Kokane Patil, Dr Parag Patil, Dr Jubina Puthen Purayil*, \\ Dr Kalpana Rajivkumar, Dr Prashant Kokane \\ *Corresponding Author \\ Dr Jubina Puthen Purayil
}

\begin{abstract}
Submental liposuction is most commonly performed aesthetic surgery procedure nowadays. Modern liposuction technique was first experimented by Giorgio Fischer and Arpad Fischer, subsequently the technique was significantly modified. In 1996, Michael Zocchi developed the ultrasonic liposuction which makes the aspiration easier. Various methods has been tried like dry method and wet method. The whole aim of the procedure is to remove the submental subcutaneous fat with minimal scar to maintain the facial contour.
\end{abstract}

Method: Prospective study of 30 cases who underwent submental liposuction for facial and neck rejuvenation. Cervicofacial liposuction was done in all cases under local anesthesia with sedation by the same surgical team. Preoperative and the postoperative photographs compared in terms of the cervicomandibular angle and patient satisfaction.

Result: Among the 30 cases, $86.6 \%$ of the cases in this study got excellent post operative result in their neck contour. 2 got post operative sagging in the submandibular and sub mental region. The result did not meet the patient expectation in one of the cases.

Conclusion: Submental liposuction is a very effective and reliable rejuvenation technique in selected patients. In this study we took the surgical outcome in terms of patient satisfaction to obtain good result.

\section{Introduction}

Liposuction is the most effective and most commonly done procedure for facial and neck rejuvenation. The most common method of surgical contouring of the obtuse neck is removal of the preplatysmal $\mathrm{fat}^{(1)}$. Submental and submandibular liposuction is the removal of fat from the subcutaneous tissue layer leading to contraction and changes in the mandibular contour. The cervical and mandibular contours considered as an important key factor in overall facial beauty. The ideal neck configuration has been described as having a cervicomental angle of 90-120 degrees with distinct mandibular border.

This neck aesthetics was started applying in 1970 by removing the subcutaneous neck fat by invasive methods ${ }^{(2)}$ and later many surgeons modified the approaches to get the good cosmetic result. Arpad and Giorgio Fischer in 1975 developed the technique of liposuction $^{(3)}$, later Illouz in 1977 introduced modified equipment's and extended methods for performing liposuction $^{(4)}$. For Liposuction in the initial period surgeons used the dry method, i.e. the fragmented unprepared fat was suctioned out. This procedure 
encountered high rate of postoperative complications.

Post operative complications of liposuction were reduced by injecting hypotonic saline into the fat before liposuctioning ${ }^{(5)}$. This was later modified as a tumescent technique, combining normal saline with local anesthesia ${ }^{(6)}$. Large volume of hypotonic saline and lignocaine as local anesthesia reduced the risk of post operative complications and changed liposuction as a daycare procedure. In this study we included the post operative evaluation and patient satisfaction, in account.

\section{Materials and Method}

This is a prospective study of 30 cases who had undergone sub mental liposuction for face and neck rejuvenation. Study was conducted during the period january 2018- September 2019 on the cases of people who are in the age group of 18-45. Inclusion Criteria: Patients demanding for cosmetic facial sculpturing, liposuction for face and neck region and people willing to join.

Exclusion Criteria: patients with traumatic disfigurement, Skin laxity, Patients having psychological or any other physical disease.

The people who satisfy our inclusion criteria are selected, explained about the procedure and evaluated for the preoperative check up and categorized according to Knize classification ${ }^{(7)}$.

Grade I: normal cervicomental angle

Grade II: mild cervicomental angle

Grade III: moderate cervicomental angle

Grade IV: severely oblique cervicomental angle ${ }^{(7)}$. All 30 cases were operated by the same surgical team. Procedure for cervicofacial liposuction was taken under local anesthesia with sedation.

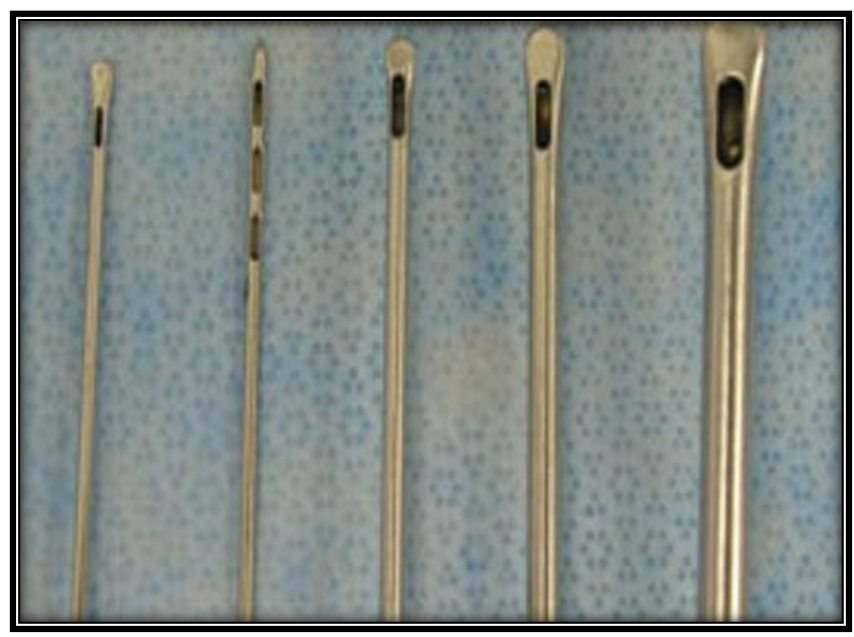

Figure 1: Suction cannula of diffent size

Markings are made for the infusion of tumescence anesthesia and aspiration of fat. Tumescence infiltration uses large volumes if diluted hypotonic solutions of vasoconstrictor agents, which is injected into the subcutaneous fat. Tumescence is made with $1 \mathrm{~L}$ ringer lactate, 2amp adrenaline, lamp hyalase, $10 \mathrm{mg}$ triamcinolone, $40 \mathrm{ml} 2 \%$ xylocaine, $20 \mathrm{ml} 0.5 \%$ bupivacaine, and $40 \mathrm{ml}$ of sodabicarbonate. Infiltration of tumescence is given with infiltration cannula of 14 gauge in subdermal plane until sufficient turgor was achieved (300-500ml) stab incision were given , for neck liposuction three stab are given, one in submental area and two below and behind each ear lobe. Fat is aspirated with suction cannula of $3 \mathrm{~mm}$ size. Post operatively compression garment of face and neck is given for five days.

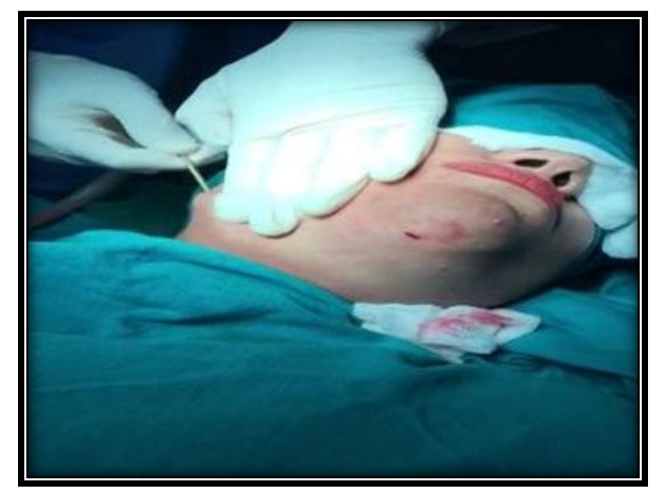

Figure 2: Liposuction Procedure

Preoperative and the postoperative photographs compared in terms of the cervicomandibular angle. All patients signed an informed consent preoperatively, and they were asked to grade their 


\section{JMSCR VoI||08||Issue||07||Page 64-67||July}

result and their skin tightening and neck-face contours postoperatively in each follow-up visit using a 5-point scale as follows: 1, poor; 2, no change; 3 , moderate; 4 , good; and 5, excellent.

\section{Results}

In our study majority of the cases good improvement in the neck contour was observed. The patient satisfaction in the post operative period was excellent, submandibular and cervicomandibular angle come out well defined.
In this study 30 patients were eligible and give consent for participation, out of which majority were females (25) and 5 males. $86.6 \%$ of the cases in this study got excellent post operative satisfaction in their neck contour. No major complications was not observed in any of the cases, however minor post operative changes like hematoma and sagging was noticed. Post operative hematoma was observed in one cases, which was treated conservatively.

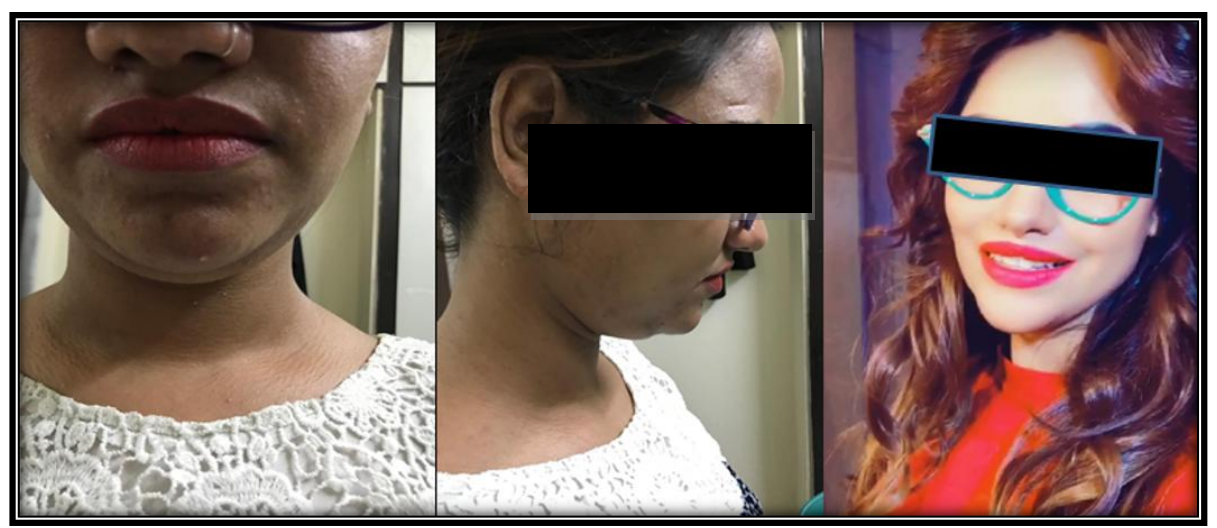

Case 1: left and middle photos are preprocedure pictures and the last one of after the procedure
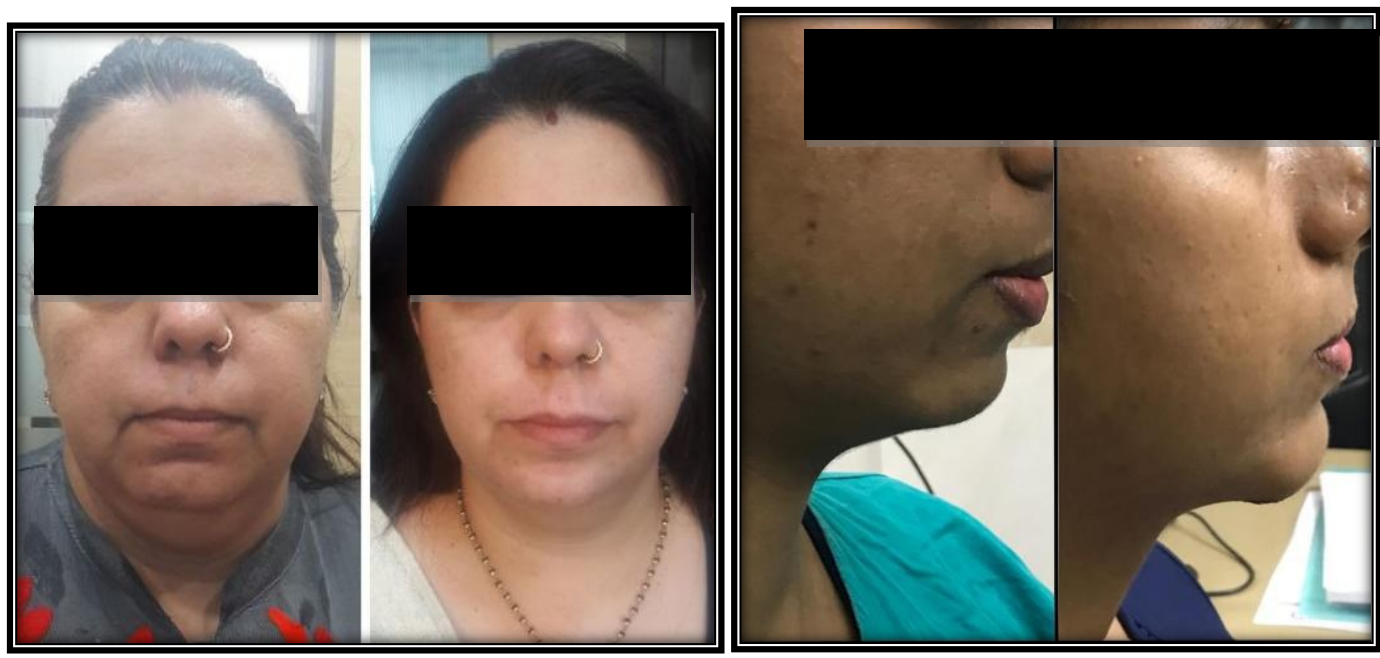

Cases: Pre procedure and post procedure pictures (right case $2 \&$ left Case 3 )

Hematoma was completely resolved after 1 month follow up period though the patient satisfaction with respect to the cervicomandibular angle was achieved well before. Among the 30 cases 2 got post operative sagging in the submandibular and sub mental region, which was treated as a second stage surgery. In one case of post operative sagging we did sub mentoplasty and the other case minifacial lift after discussing and explaining to the patient, better cosmetic result was achieved after that. The result did not meet the patient expectation in one of the cases.

\section{Discussion}

Anatomic knowledge has a major role in the enhancement of many rejuvenative procedures. Facial anatomy has progressed very much, such that we now have the detailed understanding about 
discrete fat compartment in the superficial facial fat layer ${ }^{(8)}$. This knowledge about the submental fat and its compartment aid the understanding of aging in the face, which potentially lead to better methods to treat the process of aging.

Liposuction was performed more frequently now a day with a low risk of major complications. Many studies were conducted with liposuction alone and also along with other cosmetic surgeries in combination with liposuction. Christodoulos Kaoutzanis et.al. came to a conclusion that liposuction alone give better response in terms of neck contour with less complication rate as compared with the combine procedures ${ }^{(9)}$. Alessandro Innocenti et.al. in this study they narrated about the tips and tricks for achieving good results in liposuction of the sub mental region $^{(10)}$.

The absence of the scar is the major hallmark in the liposuction technique and makes this procedure acceptable to youngsters. The unsatisfactory result may be due to the presence of deep fat below the platysma muscle or platysma band dislocation.

\section{Conclusion}

Neck liposuction is a very effective and reliable rejuvenation technique in selected patients. In this study we took the surgical outcome in terms of patient satisfaction to obtain good result. Liposuction performed under local tumescence anesthesia is an effective and safe procedure.

Patient selection based on age, anatomical features and expectations was fundamental to obtain impressive improvement of face and neck contour.

\section{Reference}

1. Hester TR Jr Douglas $\mathrm{T}$ Szczerba $\mathrm{S}$. Decreasing complications in lower lid and midface rejuvenation: the importance of orbital morphology, horizontal lower lid laxity, history of previous surgery, and minimizing trauma to the orbital septum: a critical review of 269 consecutive cases. Plast Reconstr Surg 2009;123:1037-1049.

2. L.K. Kesselring, R. Meyer, A suction curette for removal of excessive local deposits of subcutaneous fat, Plast. Reconstr. Surg. 62 (1978) 305.

3. Fischer, G. Fischer, First surgical treatment for molding body's cellulite with three $5 \mathrm{~mm}$ incisions, Bull. Int. Acad. Cosmet. Surg. 3 (1976) 35.

4. Y. Illouz, Body contouring by lipolysis: a 5 year experience with over 3000 cases, Plast. Reconstr. Surg. 72 (1983) 511.

5. Klein JA: The tumescent technique for liposuction surgery. Am J Cosmetic Surg 4:236-267, 1987.

6. Rohrich RJ, Pessa JE. The retaining system of the face: histologic evaluation of the septal boundaries of the subcutaneous fat compartments. Plast Reconstr Surg 2008; 121:1804-1809.

7. Knize DM (1998) Limited incision submental lipectomy and platysmaplasty. Plast Reconstr Surg 101:473-481

8. Christodoulos Kaoutzanis, Varun Gupta, Julian Winocour, John Layliev, Roberto Ramirez, James C. Grotting, Kent Higdon, Cosmetic Liposuction: Preoperative Risk Factors, Major Complication Rates, and Safety of Combined Procedures, Aesthetic Surgery Journal, Volume 37, Issue 6, 1 June 2017, Pages 680-694.

9. Alessandro Innocenti, Chiara Andretto Amodeo, Francesco Ciancio, Wideundermining Neck Liposuction: Tips and Tricks for Good Results, Aesth Plast Surg, 2014 Aug;38(4):662-9.

10. Knize DM (1998) Limited incision submental lipectomy and platysmaplasty. Plast Reconstr Surg 101:473-481. 